\title{
Portable Device for Skin Cancer Diagnostics by Combined Diffuse Reflectance and Autofluorescence Photobleaching
}

\author{
Ilona Kuzmina ${ }^{1}$, Dmitrijs Bliznuks ${ }^{2}$, Vanesa Lukinsone ${ }^{1}$, Emilija Vija Plorina ${ }^{1}$, Janis Spigulis ${ }^{1}$ \\ ${ }^{1}$ Institute of Atomic Physics and Spectroscopy, University of Latvia \\ Raina blvd. 19, Riga, Latvia \\ ilona.kuzmina@1u.lv; vanesa.lukinsone@lu.lv; evplorina@gmail.com; janis.spigulis@1u.lv \\ ${ }^{2}$ Faculty of Computer Science and Information Technology, Riga Technical University \\ Daugavgrivas str. 2, Riga, Latvia \\ dmitrijs.bliznuks@rtu.lv
}

\section{Extended Abstract}

Since an incidence of skin cancer is still a topical issue in Europe [1,2], the aim of this work is to develop a low-cost user-friendly portable device for skin cancer screening in general practitioner practice that could significantly improve the accessibility of early diagnostics, thereby reducing mortality. Previously developed algorithm by using visible and near infrared diffuse reflectance images at selected wavelengths showed high sensitivity and specificity for melanoma diagnostics [3]. A temporal analysis of autofluorescence intensity during the photobleaching showed a potential for primary evaluation of basal cell carcinoma [4].

The portable device has been designed for melanoma and non-melanoma cancer diagnostics by using combined diffuse reflectance and autofluorescence photobleaching imaging at selected wavelengths. The device consists of a ring of green $(540 \mathrm{~nm})$, red $(650 \mathrm{~nm})$ and infrared $(950 \mathrm{~nm})$ LEDs for diffuse reflectance imaging and violet $(405 \mathrm{~nm})$ LEDs for autofluorescence photobleaching imaging. Skin images are acquired by colour CMOS sensor (IDS 5MPix) in combination with cross polarized narrowband LEDs illumination. Raspberry Pi Compute Module performs data acquisition and processing. The device includes WiFi and $4 \mathrm{G}$ cellular modems for a wireless transfer of the images for further image processing at cloud computing service and remote access to the diagnostic results.

The first results of combination of diffuse reflectance and autofluorescence imaging showed a promising differentiation of neoplasms that could be used for skin cancer diagnostics [5]. The temporal imaging of autofluorescence intensity that can be performed by this device will bring additional improvements in diagnostic accuracy. By comparing proposed method with machine learning algorithms, we can expect slightly better diagnostic quality, but the most important difference is the ability to start diagnostic procedure on virtually any hardware without the need to implement learning phase, that requires huge set of images.

\section{References}

[1] J. Ferlay, E. Steliarova-Foucher, J. Lortet-Tieulent, S. Rosso, J. W. W. Coebergh, H. Comber, D. Forman and F. Bray, "Cancer incidence and mortality patterns in Europe: Estimates for 40 countries in 2012," Eur. J. Cancer., vol. 49, no. 6, pp. 1374-1403, 2013.

[2] European Commission, (2018, May 24). Eurostat statistics explained: Cancer statistics. [Online]. Available: http://ec.europa.eu/eurostat/statistics-explained/index.php/Cancer_statistics

[3] I. Diebele, I. Kuzmina, A. Lihachev, J. Kapostinsh, A. Derjabo, L. Valeine and J. Spigulis, "Clinical evaluation of melanomas and common nevi by spectral imaging," Biomedical Optics Express, vol. 3, no. 3, pp. 467-472, 2012.

[4] A. Lihachev, A. Derjabo, I. Ferulova, M. Lange, I. Lihacova and J. Spigulis, "Autofluorescence imaging of basal cell carcinoma by smartphone RGB camera,” J. Biomed. Opt., vol. 20, no. 12, pp. 120502-1-4, 2015.

[5] I. Lihacova, K. Bolochko, E. V. Plorina, M. Lange, A. Lihachev, D. Bliznuks and A. Derjabo, "A method for skin malformation classification by combining multispectral and skin autofluorescence imaging," in Proceedings of SPIE, vol. 10685, pp.1068535-1-9, 2018. 